Supplement of Hydrol. Earth Syst. Sci. Discuss., 12, 12515-12566, 2015

http://www.hydrol-earth-syst-sci-discuss.net/12/12515/2015/

doi:10.5194/hessd-12-12515-2015-supplement

(C) Author(s) 2015. CC Attribution 3.0 License.

(c) (1)

Hydrology and

\title{
Estimating drought risk across Europe from reported drought impacts, hazard indicators and vulnerability factors
}

\section{Blauhut et al.}

Correspondence to: V. Blauhut (veit.blauhut@hydrology.uni-freiburg.de)

The copyright of individual parts of the supplement might differ from the CC-BY 3.0 licence. 


\section{Supplements}

3 Table S1, European countries with insufficient vulnerability data

4 Albania

Armenia

Azerbaijan

Bosnia and Herzegovina

Belarus

Georgia

Moldavia

Montenegro

Macedonia

Serbia

Russia

Slovenia

Turkey 
1 Table S 2 Results of binary logistic model performance (step one), drought indices used in EDO

2 (2001-2014), I: Maritime Europe, II: Southeastern Europe, III: Northeastern Europe, IV:

3 Western-Mediterranean; white coloring + no '*': no robust model identified, light green

4 coloring + '*': 'poor' model performance, green coloring + '**': 'good' model performance,

5 dark green coloring + '***': 'excellent' model performance

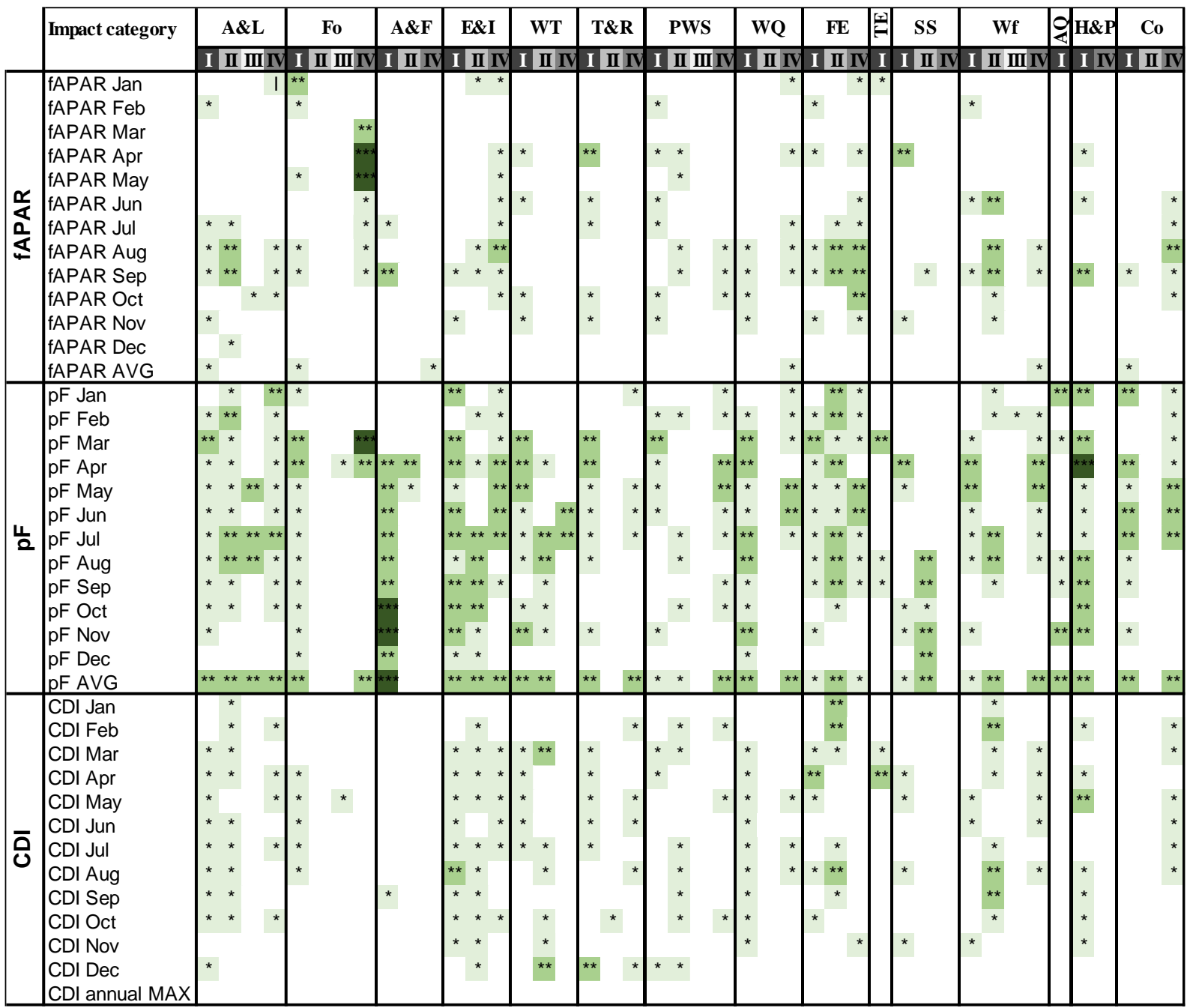

10 Table S 3 Results of binary logistic model performance (step one), SPEI 1, 3, 6, 12, 24; (197011 2012), I: Maritime Europe, II: Southeastern Europe, III: Northeastern Europe, IV: Western12 Mediterranean, white coloring + no '*': no robust model identified, light green coloring + '*': 13 'poor' model performance, green coloring + '**': 'good' model performance, dark green 14 coloring + '***': 'excellent' model performance 


\begin{tabular}{|c|c|c|c|c|c|c|c|c|c|c|c|c|c|c|}
\hline \multirow{2}{*}{\begin{tabular}{|l|}
$\begin{array}{l}\text { Impact } \\
\text { category }\end{array}$ \\
\end{tabular}} & $\mathbf{A} \& \mathbf{L}$ & Fo & $A \& F$ & E\&I & WT & T\&R & PWS & WQ & FE & 国 & Wf & \multicolumn{2}{|c|}{$H \& P$} & Co \\
\hline & & & & I I II & I II I & & I II IIIIV & I II I & & & I II III & & & II IV \\
\hline SPEI-01 Jan & & & & & & & & & & & & & & ** \\
\hline SPEI-01 Feb & & *** & & ** & * & * & * * & & * & ** & & & *夫 & \\
\hline SPEI-01 Mar & & ** & ** & * * & ** & ** & * * & * * & * & ** * & * * & $* *$ & ** & \\
\hline SPEI-01 Apr & $\mid \begin{array}{l}* \\
*\end{array}$ & * & & * & & & $\begin{array}{l}* * \\
* *\end{array}$ & * & & * & $* * * *$ & & ** & ${ }_{* \star}^{* *}$ \\
\hline SPEI-01 Jun & ***** & & & ** * & & * & & ** & & **| * & **** & & *** & \\
\hline SPEI-01 Jul & ** & & & & & & ** * & * & & * & $* * *$ & & * & \\
\hline SPEI-01 Aug & & * & ** & **** & & & ** * & * & * * & 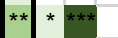 & $\star \star \star \star \star ~$ & & ** & ** \\
\hline SPEI-01 Sep & & * & & & * & & * & & & & & ** & & \\
\hline $\begin{array}{l}\text { SPEI-01 Oct } \\
\text { SPEI-01 Nov }\end{array}$ & & * & * & * * & *** & * & & * & * & ${ }_{*}^{*}$ & & & * & \\
\hline SPEI-01 Dec & & & & & & * & & & & & & & & \\
\hline SPEI-03 Jan & & & & & & * & * & * & & * & ** & * & * & \\
\hline SPEI-03 Feb & ${ }_{* \star *}^{*}$ & ${ }_{*}^{*}$ & & & ${ }^{*}$ & ${ }^{*}$ & * & & * & ***** & $*$ & & * & \\
\hline SPEI-03 Apr & ** * & ** & & & ** & ** & * * & ** & * & **** & ** & & & \\
\hline SPEI-03 May & & ** & ** & ** & & ** & ** & & & & & 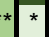 & ** & \\
\hline SPEI-03 Jun & & & *** & & & & & & & ${ }_{* \star * * * *}^{* *}$ & & & ** & \\
\hline $\begin{array}{l}\text { SPEI-03 Jul } \\
\text { SPEI-03 Aut }\end{array}$ & & & & & & & $\begin{array}{ll}* & * \\
* & *\end{array}$ & & & & & & *夫 & \\
\hline SPEI-03 Sep & & & ** & **** & *** & & * * & & & * & **** & & ** & ** \\
\hline SPEI-03 Oct & & *** & ** & & & & ** * & * * & & & & & & \\
\hline SPEI-03 Nov & * & * & * & * & * & * & * & * * & & & & ** & * & \\
\hline SPEI- & * & * & & * & & & & * & & & & & & \\
\hline SPEI-0 & * & 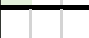 & & ${ }^{*} *$ & & & ${ }_{* *}^{*}$ & & & & ** & & & \\
\hline SPEI-06 Feb & & & & & * & * & * * & & & & & & & \\
\hline SPEI-06 Mar & & * & & & * & ** & ** * & & & * & *** & & & \\
\hline $\begin{array}{l}\text { SPEI-06 Apr } \\
\text { SPEI-06 Mav }\end{array}$ & $\left.\right|_{* \star *} ^{*} *$ & & & & ** & *** & $\mid \begin{array}{l}* \\
*\end{array}$ & & & ${ }^{*}$ & & & ${ }^{*}$ & \\
\hline SPEI-06 Jun & & ** * & & & ** & ** & $* * *$ & & & ** & & ${ }^{*}$ & ** & \\
\hline SPEI-06 Jul & & & & & & ** & ${ }^{* * * *}$ * & & & ** & 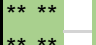 & $t_{ \pm}^{*}$ & *** & \\
\hline $\begin{array}{l}\text { SPEI-06 Aug } \\
\text { SPEI-06 SeD }\end{array}$ & & & & & & & $* * *$ & ** & & & 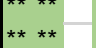 & $x_{* \star *}$ & *** & \\
\hline SPEI-06 Oct & & ** & & **** & & * & & ** & & & ** * & & ** & \\
\hline SPEI-06 Nov & $\mid * * * \star$ & ** & $\begin{array}{l}* \star \\
\star \star\end{array}$ & $\begin{array}{c}* * * * \\
* * *\end{array}$ & $\begin{array}{l}* * * * \\
* *\end{array}$ & * & $\begin{array}{ll}* & * \\
* & *\end{array}$ & ${ }_{* *}^{* *} *$ & ${ }^{* * * *}$ & ${ }^{*} *$ & 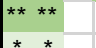 & ** & ** & \\
\hline \begin{tabular}{|l} 
SPEI-06 Dec \\
SPEI-12 Jan
\end{tabular} & $* *$ & & & & & & $* *$ & $* *$ & & & & & & \\
\hline SPEI-12 Feb & * & & & ** * & * & * & ** * & * * & & * & $* *$ & & & ** \\
\hline SPEI-12 Mar & & * & & & * & ** & ** * & $* * *$ & & & *** & & & \\
\hline SPEI-12 Apr & & & & & * & ** & ** & & & * & $* * *$ & & & \\
\hline SPEI-12 May & & * & & & ** & ** & * * & & & * & *** & & & \\
\hline SPEI-12 Jun & * ** $\quad$ * & * * & & **** & ** & ** & * * & ** & & & *** & & & \\
\hline SPEI-12 Jul & 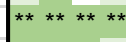 & ** & & **** & ** & ** & * * & ** & & & 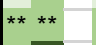 & & * & \\
\hline SPEI-12 Aug & & & & & & 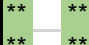 & ${ }^{*} * *$ & & & ${ }_{* * * *}^{*}$ & 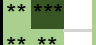 & * & 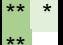 & \\
\hline 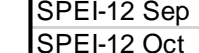 & & **** & & & & *** & $x_{* \star *}^{*}$ & ****** & & 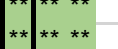 & ***** & & ** & \\
\hline & & ** & & & & ** & & & & & **** & $*$ & ** & \\
\hline SPEl-1 & & *夫 & & & 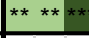 & 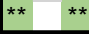 & ** * & & & 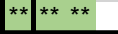 & $\star \star \star \star \star *$ & & ** & \\
\hline SPEI-24 Jan & & * & & & & * & & & & & & & & \\
\hline SPEI-24 Feb & * & & & & * & * & * * & & & * & & * & & ** * \\
\hline $4 \mathrm{Mar}$ & & & & & & ** & & & & & & & & \\
\hline SPEI-24 Apr & & & & & * & ** & ** & & * & * & * & & & \\
\hline SPEI-24 May & & & & & * & ** & ** & & & * & & & & \\
\hline SPEI-24 Jun & & * & & & & ** & ** & & & * & * * & & & ** * \\
\hline SPEI-24 Jul & & & & & & ** & **** & & & & *** & & & \\
\hline SPEI-24 Aug & * & & & & * & ** & & & & * & 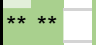 & & & \\
\hline SPEI-24 Sep & & * & *** & ** & ** & ** & *** * & & & * & **** & * & & \\
\hline EI-24 Oct & **** & ** & * & ***** & ** ** & ** & & 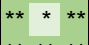 & 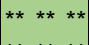 & **** & **** & * & & \\
\hline & $\mid \begin{array}{l}* \star * * \\
* * * *\end{array}$ & *** * & $\mid \begin{array}{l}* * * * \\
* * * *\end{array}$ & $\begin{array}{l}\star \star \star \star \star \star * \\
\star \star \star \star \star *\end{array}$ & $\begin{array}{l}* * * * * \\
* * * *\end{array}$ & ** & $\begin{array}{l}* * * * \\
* * * *\end{array}$ & 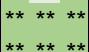 & 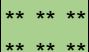 & $\left|\begin{array}{c}\star \star \\
\star \star\end{array}\right| \begin{array}{l}* * \\
*\end{array}$ & $\mid \begin{array}{l}* \star \star * \star \\
* \star \star \star *\end{array}$ & & ** & \\
\hline SPEI-24 Dec & $I^{* * \star *} \quad \star * *$ & & $D^{* * * *}$ & 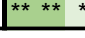 & **** & ** $\quad$ ** & \begin{tabular}{|l|l}
$* \star \star *$ & $\star$ \\
\end{tabular} & ****** & ****** & |*x* & **** & & & *夫 \\
\hline
\end{tabular}


23 Table S 4 Results of binary logistic model performance (step one), vulnerability factors, I: 24 Maritime Europe, II: Southeastern Europe, III: Northeastern Europe, IV: Western25 Mediterranean; A. = Area of, dens. = density, EC $=$ European Commission, GDP = Gross 26 Domestic Product, GW = Groundwater, norm. = normalised, NC = NUTS-combo region, N2 = 27 NUTS-2, SR = Socioeconomic Relevance, WR= Water Use Relevance 


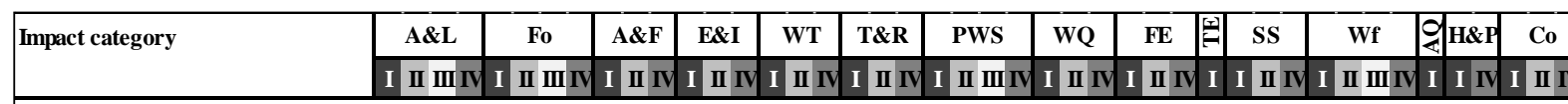

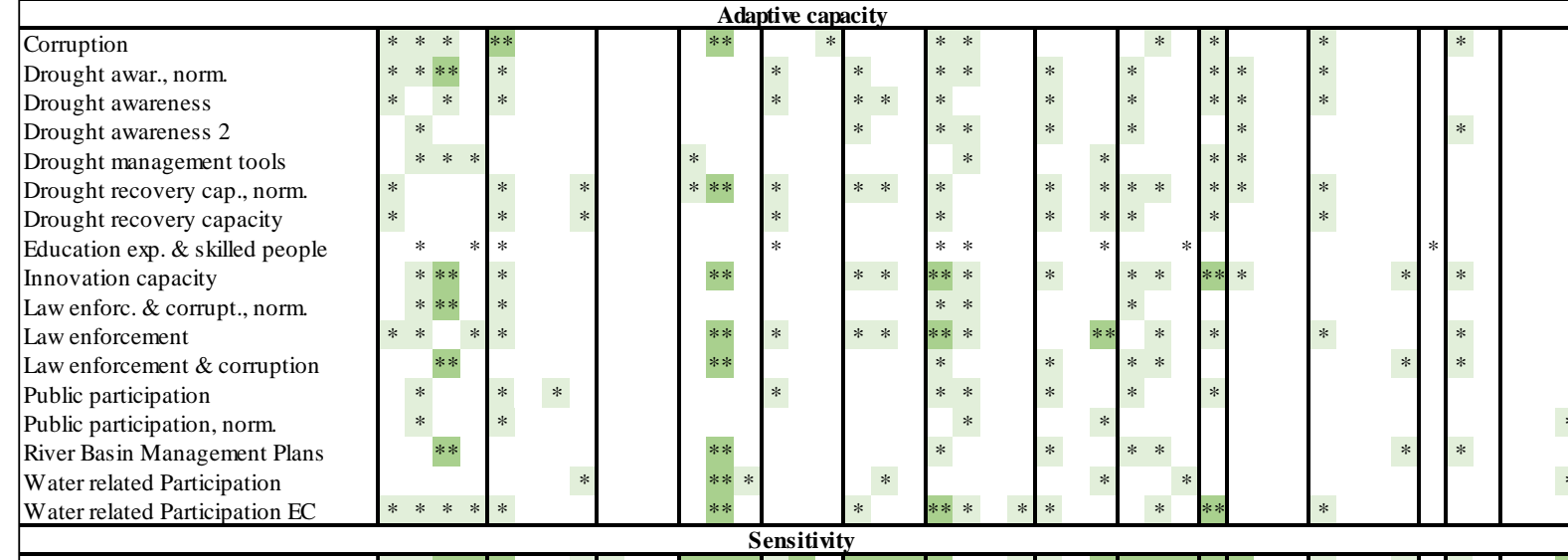

A agriculture

A. agriculture, ratio of $\mathrm{NC}$

A. artificial surfaces

A. artificial surfaces, ratio of $\mathrm{NC}$

A. forest

A. forest, ratio of $\mathrm{NC}$

A. inland water bodies

A. inland water bodies, ratio of NC

A. lakes within region

A. non irrigated agri.

A. non irrigated agri, ratio of NC

A. perm. irrigated. agri.

A. semi natural areas

A. semi natural areas, ratio of NC

A. wetlands

A. wetlands, ratio of $\mathrm{NC}$

Aquatic ecosystem status

Arable land

A. NUTS-combo region

Biodiversity, A. protect

Dams + GW resources

Dams + GW resources, norm.

Dams capacity 2

Dams resources, norm.

Economic resources \& equity

Economic wealth

Education

Environmental taxes

GDP by country

Groundwater resources (GW)

Groundwater resources, norm.

Human health \& public safety

Population density N2

Population dens. \& age, norm.

Population dens. \& age.

Population dens. by country

PWS by N2

PWS connection by N2

Soil type

SR Agri., norm.

SR agriculture

SR indus

SR industry, norm

SR services sector

SR services sector, norm.

Tourist beds by country

Tourist beds by N2

Water balance

Water body status

Water exploitation index

Water exploitation index, norm

Water resources development

Water use

Water use Agri

Water use Indus

WR agri sector

WR agri., norm.

WR industry sector

WR industry sector, norm

WR services sector

WR services sector 2

WR services sector, norm.
WR

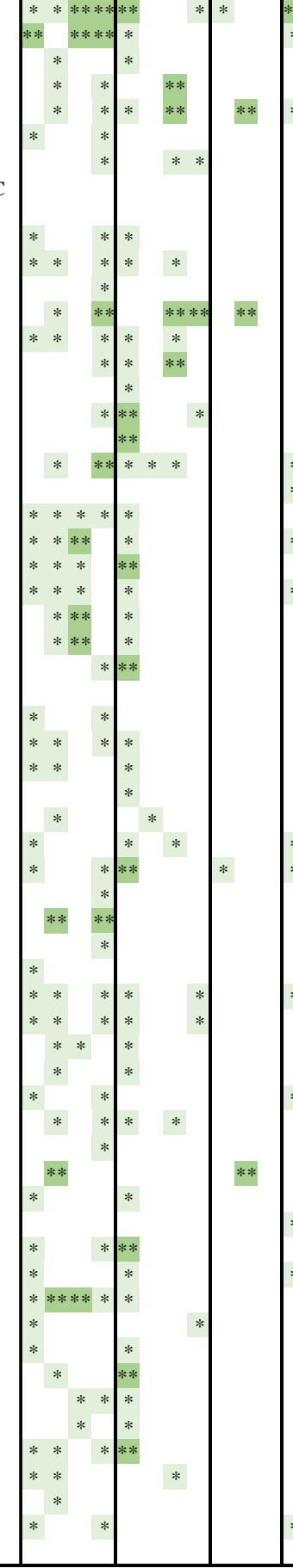

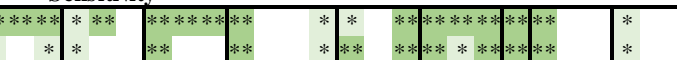

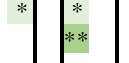

\section{.}

Combined factors

\begin{tabular}{|l|l|l|l|l|l|}
\hline SENSITIVITY & $*$ & $*$ & $*$ & & $* *$ \\
\hline
\end{tabular} 